6-2012

\title{
Predictive Musculoskeletal Simulation Using Optimal Control: Effects of Added Limb Mass on Energy Cost and Kinematics of Walking and Running
}

Antonie J. van den Bogert

a.vandenbogert@csuohio.edu, a.vandenbogert@csuohio.edu

Maarten Hupperets

$\operatorname{adidas} A G$

Heiko Schlarb

adidas $A G$

Berthold Krabbe

$\operatorname{adidas} A G$

Follow this and additional works at: https://engagedscholarship.csuohio.edu/enme_facpub

Part of the Biomechanical Engineering Commons

How does access to this work benefit you? Let us know!

\section{Original Citation}

van den Bogert AJ, Hupperets M, Schlarb H, Krabbe B (2012) Predictive musculoskeletal simulation using optimal control: Effects of added limb mass on energy cost and kinematics of walking and running. Proc Inst Mech Eng, Part P: J Sports Eng Technol 226: 123-133.

This Article is brought to you for free and open access by the Mechanical Engineering Department at EngagedScholarship@CSU. It has been accepted for inclusion in Mechanical Engineering Faculty Publications by an authorized administrator of EngagedScholarship@CSU. For more information, please contact library.es@csuohio.edu. 


\title{
Predictive musculoskeletal simulation using optimal control: effects of added limb mass on energy cost and kinematics of walking and running
}

Proc IMechE Part P:

J Sports Engineering and Technology 226(2) 123-133

(c) IMechE 2012

Reprints and permissions:

sagepub.co.uk/journalsPermissions.nav DOI: 10.1 I $77 / 1754337$ | I 2440644

pip.sagepub.com

@SAGE

\author{
Antonie J van den Bogert ', Maarten Hupperets ${ }^{2}$, Heiko Schlarb ${ }^{2}$ and \\ Berthold Krabbe ${ }^{2}$
}

\begin{abstract}
When designing sports equipment, it is often desirable to predict how certain design parameters will affect human performance. In many instances, this requires a consideration of human musculoskeletal mechanics and adaptive neuromuscular control. Current computational methods do not represent these mechanisms, and design optimization typically requires several iterations of prototyping and human testing. This paper introduces a computational method based on musculoskeletal modeling and optimal control, which has the capability to predict the effect of mechanical equipment properties on human performance. The underlying assumption is that users will adapt their neuromuscular control according to an optimality principle, which balances task performance with a minimization of muscular effort. The method was applied to the prediction of metabolic cost and limb kinematics while running and walking with weights attached to the body. A two-dimensional musculoskeletal model was used, with nine kinematic degrees of freedom and 16 muscles. The optimal control problem was solved for two walking speeds and two running speeds, and at each speed, with $200 \mathrm{~g}$ and $400 \mathrm{~g}$ masses placed at the thigh, knee, shank and foot. The model predicted an increase in energy expenditure that was proportional to the added mass and the effect was largest for a mass placed on the foot. Specifically, the model predicted an energy cost increase of $0.74 \%$ for each $100 \mathrm{~g}$ mass added to the foot during running at $3.60 \mathrm{~m} / \mathrm{s}$. The model also predicted that stride length would increase by several millimetres in the same condition, relative to the model without added mass. These predictions were consistent with previously published human studies. Peak force and activation remained the same in most muscles, but increased by $26 \%$ in the hamstrings and by $17 \%$ in the rectus femoris for running at $4.27 \mathrm{~m} / \mathrm{s}$ with $400 \mathrm{~g}$ added mass at the foot, suggesting muscle-specific training effects. This work demonstrated that a musculoskeletal model with optimal control can predict the effect of mechanical devices on human performance, and could become a useful tool for design optimization in sports engineering. The theoretical background of predictive simulation also helps explain why human athletes have specific responses when exercising in an altered mechanical environment.
\end{abstract}

\section{Keywords}

Running, simulation, musculoskeletal modeling, optimal control

\section{Introduction}

Well-designed sports equipment has the potential to improve performance and prevent injuries. Often, however, these products do not work as expected. A wellknown example is the failure of soft-soled shoes to reduce impact forces during running, ${ }^{1}$ possibly due to complex nonlinear mechanics of the musculoskeletal system, ${ }^{2}$ as well as the user's adaptive behavior. ${ }^{3-4}$ Computer models of sports equipment are increasingly used in the design process, but these do not include the musculoskeletal or behavioral mechanisms. Consequently, the process of testing of equipment prototypes in actual users remains an essential and time consuming

\footnotetext{
'Orchard Kinetics, USA

${ }^{2}$ ait Sport Research Laboratory, adidas AG, Germany

Corresponding author:

Antonie J van den Bogert, Orchard Kinetics LLC, 2217 South Overlook Road, Cleveland, OH 44I06, USA.

Email: bogert@orchardkinetics.com
} 
step in the design optimization process. Computational musculoskeletal models may offer an opportunity to predict certain aspects of performance prior to the prototyping stage, but existing models require that muscle activation patterns are fully controlled by the experimenter. This has limited their application to brief motion segments and to low-level questions. ${ }^{2,5}$ In this paper, a new task-level optimal control approach is presented in which a musculoskeletal model generates its own muscle activations in order to optimally perform a given movement task. With this approach, a model can be used similarly to a human research participant: equipment parameters are altered, and the resulting change in performance is quantified. The concept will be demonstrated by using a musculoskeletal model to predict the effect of added limb mass on walking and running. This topic is relevant to many sports and has been studied extensively in human participants, thus allowing an evaluation of how effective this general predictive modeling approach is in predicting actual human performance.

Mass distribution in the lower extremity is an important determinant of performance in running. In cursorial animals, which have evolved to be specialized for running, most muscle mass is concentrated near the proximal parts of the limb, presumably to reduce the moment of inertia of the limb and allow rapid forward acceleration during the swing phase. ${ }^{6}$ Similarly, in human running, the mass of the footwear (typically $0.35 \mathrm{~kg}$ per shoe) is reduced as much as possible in order to maximize performance. ${ }^{7}$ Low mass is also thought to be responsible for the remarkable running performance recently observed in a transtibial amputee. ${ }^{8}$ In view of these well-known effects of limb mass on performance, it is of interest to consider whether an increase in limb mass and moment of inertia might have a desirable training effect. These parameters are easily manipulated by attaching discrete masses at specific points on the limb. Such training may help athletes perform better during competition when the additional mass is removed.

Most research on the effect of limb mass has been focused on the metabolic energy cost of added masses, while running at a given speed. The increase in metabolic cost was found to be proportional to the amount of added mass, ${ }^{9-11}$ and dependent on its location. For masses placed on the foot and ankle, the increase in metabolic cost ranged from $0.7 \%$ to $1.3 \%$ per $100 \mathrm{~g}$ of added mass, depending on running speed and subject population. ${ }^{9-13}$ Metabolic effects of mass placed more proximally were between $0.3 \%$ and $0.7 \% .^{10-12}$ These metabolic effects are too small to be easily detected by measuring oxygen uptake, but large enough to determine who wins a race. Since metabolic cost is proportional to running speed, ${ }^{14}$ a lower metabolic cost at a given speed translates directly into the ability to run faster while maintaining the same metabolic steady state (e.g. $80 \%$ of maximal oxygen uptake). One study reported a mechanical effect of added limb mass during running: stride length was increased by $1.4 \mathrm{~cm}$, and swing time by $9 \mathrm{~ms}$, when a $0.5 \mathrm{~kg}$ mass was attached to each foot. ${ }^{11}$ More detailed biomechanical measures, such as joint angles, ground reaction forces and muscle activations, have not been reported for running with added weights. These mechanical effects, while potentially important for training and performance, are expected to be rather subtle and not statistically significant in a typical movement analysis protocol with its inevitable random variability. ${ }^{15}$

When considering the use of attached mass for training purposes, the design parameters are the amount of mass, and where it is attached to the limb. Multiple masses are possible. This entire multidimensional parameter space must be explored in order to achieve the maximal desired training effect with minimal side effects. Of specific interest are the mechanical effects, such as increased recruitment of specific muscles, which would represent targeted training. Direct measurement of muscle recruitment via EMG suffers from poor reproducibility and can only detect large effects. ${ }^{16}$ Indirect methods, such as inverse dynamic analysis have been presented recently, ${ }^{17}$ but these require an assumption of optimal muscle recruitment (similar to what will be used in this paper) as well as data collection on human participants exposed to each of the mechanical test conditions. Any design optimization via human testing will be costly, technically difficult, and may require excessively large sample sizes and test protocols in order to achieve statistical significance when the mechanical changes are subtle. Predictive computational modeling, which will be presented and evaluated in this paper, would reduce the need for testing of prototypes on human participants.

The purpose of this paper is threefold: (i) to describe a novel optimal control approach for predictive musculoskeletal simulation, (ii) to apply this method to the prediction of energy cost and movement during running with added limb weights, and (iii) to compare the results of the computational model to results from previous human studies.

\section{Methods}

\section{Musculoskeletal model}

The musculoskeletal model was two dimensional and based on an early model of running ${ }^{18}$ that was subsequently modified for applications in skiing, ${ }^{19}$ vehicle accidents, $^{20}$ and walking. ${ }^{21,22}$ The model has seven body segments, six joints (hip, knee and ankle in each limb), nine kinematic degrees of freedom, and 16 muscles (Figure 1). Inertial properties of limb segments for a typical adult male were obtained from the literature. $^{23}$ Added mass was represented by an increased segment mass, a shift in segment center of mass, and an increased moment of inertia based on the location of the mass and the parallel axes theorem. Changes were always the same in the left and right limbs, keeping the 


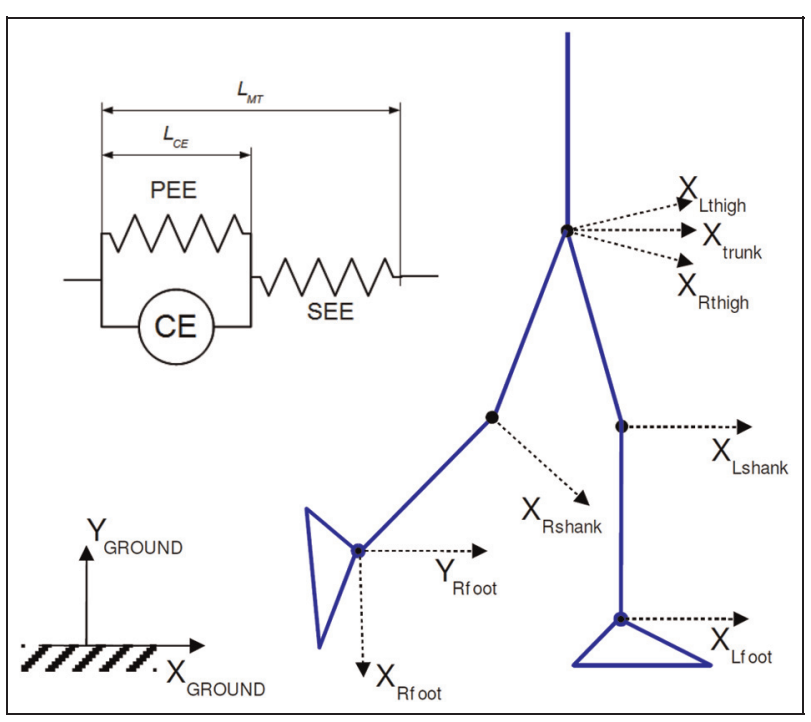

Figure I. The skeletal model with local reference frames for each of the seven body segments (right) and the three-element muscle model that was used to generate movement (top left). CE: contractile element; PEE: parallel elastic element; SEE: series elastic element.

model perfectly symmetrical. Contact between foot and ground was represented by unidirectional visco-elastic elements with friction, as described previously. ${ }^{22}$ Equations of motion were derived with Autolev (Online Dynamics, Sunnyvale, CA) with respect to the nine generalized coordinates $\mathbf{q}$

$$
\mathbf{M}(\mathbf{q}) \cdot \ddot{\mathbf{q}}+\mathbf{B}(\mathbf{q}, \dot{\mathbf{q}})-\boldsymbol{\tau}=0
$$

where $\mathbf{M}(\mathbf{q})$ is the mass matrix and $\mathbf{B}(\mathbf{q}, \dot{\mathbf{q}})$ contains gravity, centrifugal and coriolis effects, as well as passive joint moments and contact forces, which are modeled as a function of generalized coordinates and velocities. The vector $\tau$ contains the nine generalized forces, of which the first three are zero because global trunk position and orientation are unactuated. The remaining six generalized forces are the joint moments, which are related to muscle forces:

$$
\left(\begin{array}{c}
\tau_{4} \\
\vdots \\
\tau_{9}
\end{array}\right)=\mathbf{R F}
$$

where $\mathbf{R}$ is a $6 \times 16$ matrix containing the moment arms Rij of muscle $i$ at joint $j$. Muscle forces $\mathbf{F}$ were generated by a conventional three-element Hill-based model (Figure 1) with activation dynamics, force-length properties, and force-velocity properties. ${ }^{24}$ This introduces two differential equations for each muscle to describe contraction dynamics and activation dynamics, with the corresponding state variables being the contractile element length $L_{\mathrm{CE}}$ and the active state $a .{ }^{25}$ Muscle model parameters are shown in in Table 1.

This musculoskeletal system model has 50 state variables: generalized coordinates and velocities for each of the nine degrees of freedom, plus contractile element length and active state for each of the 16 muscles. These are collected in a state vector $\mathbf{x}$

$$
\mathbf{x}=\left(\mathbf{q}, \dot{\mathbf{q}}, \mathbf{L}_{C E}, \mathbf{a}\right)^{T}
$$

The model is driven by 16 control inputs, the neural excitations for the muscles, which are collected in a control vector $\mathbf{u}$. The system dynamics, which consists of the coupled multibody dynamics and muscle dynamics, can now be formulated as an implicit differential equation $^{26}$

$$
\mathbf{f}(\mathbf{x}, \dot{\mathbf{x}}, \mathbf{u})=0
$$

The function $\mathbf{f}$ was coded in $\mathrm{C}$, with a Matlab MEX function interface so the model could be used with Matlab (Mathworks, Natick MA). The MEX function also calculated the sparse analytical Jacobian matrices $\partial \mathbf{f} / \partial \mathbf{x}, \partial \mathbf{f} / \partial \dot{\mathbf{x}}$, and $\partial \mathbf{f} / \partial \mathbf{u}$, which are needed in gradientbased optimal control methods. ${ }^{26}$

\section{Human movement data}

Data collection was performed on a male participant. Informed written consent in accordance with adidas AG legal counsel was obtained. Speeds (Table 2) up to

Table I. Muscle properties in the musculoskeletal model: maximal isometric force $\left(F_{\text {max }}\right)$, fiber length at maximal isometric force

\begin{tabular}{|c|c|c|c|c|c|c|c|c|}
\hline \multirow[t]{2}{*}{ Muscle } & \multicolumn{4}{|c|}{ Muscle mechanics } & \multicolumn{4}{|c|}{ Muscle-skeleton coupling } \\
\hline & $F_{\max }(\mathrm{N})$ & $L_{\text {CEopt }}(\mathrm{m})$ & $L_{\text {slackPEE }} / L_{\text {CEopt }}$ & $L_{\text {slackSEE }}(\mathrm{m})$ & $L_{0}(\mathrm{~m})$ & $d_{h i p}(m)$ & $d_{\text {knee }}(m)$ & $d_{\text {ankle }}(\mathrm{m})$ \\
\hline Iliopsoas & 1500 & 0.102 & 1.200 & 0.142 & 0.248 & 0.050 & 0 & 0 \\
\hline Glutei & 3000 & 0.200 & 1.200 & 0.157 & 0.271 & -0.062 & 0 & 0 \\
\hline Hamstrings & 3000 & 0.104 & 1.200 & 0.334 & 0.383 & -0.072 & -0.034 & 0 \\
\hline Rectus & 1200 & $0.08 I$ & 1.400 & 0.398 & 0.474 & 0.034 & 0.050 & 0 \\
\hline Vasti & 7000 & 0.093 & 1.400 & 0.223 & 0.271 & 0 & 0.042 & 0 \\
\hline Gastrocnemius & 3000 & 0.055 & 1.200 & 0.420 & 0.487 & 0 & -0.020 & -0.053 \\
\hline Soleus & 4000 & 0.055 & 1.200 & 0.245 & 0.284 & 0 & 0 & -0.053 \\
\hline Tibialis anterior & 2500 & 0.082 & 1.200 & 0.317 & 0.381 & 0 & 0 & 0.037 \\
\hline
\end{tabular}
$\left(L_{C E o p t}\right)$, slack length of the parallel and series elastic elements $\left(L_{\text {slackPEE }}, L_{\text {slackSEE }}\right)$, muscle-tendon length at neutral skeleton pose $\left(L_{O}\right)$, and the moment arms $(d)$ at each joint. Full details of the muscle model and definitions of these model parameters can be found elsewhere. $^{26}$ 
Table 2. Summary of human movement data that were used. Slow walking data were obtained from the literature. ${ }^{23}$ Speed and gait cycle duration for the other three movements are the mean and standard deviation of five trials, collected from a single male participant.

\begin{tabular}{lll}
\hline Movement & Speed $(\mathrm{m} / \mathrm{s})$ & Gait cycle duration $(\mathrm{s})$ \\
\hline Slow walking & 1.10 & 1.28 \\
Fast walking & $1.82 \pm 0.06$ & $0.96 \pm 0.02$ \\
Slow running & $3.60 \pm 0.08$ & $0.71 \pm 0.04$ \\
Fast running & $4.27 \pm 0.08$ & $0.68 \pm 0.01$ \\
\hline
\end{tabular}

$4.3 \mathrm{~m} / \mathrm{s}$ were selected based on typical speeds used for exercise. For fast walking, the target speed was set at $1.8 \mathrm{~m} / \mathrm{s}$. After five familiarization trials, the participant was asked to perform additional trials at $1.8 \mathrm{~m} / \mathrm{s}$ for data collection. Speed was measured using a set of light gates (Leuze Electronics GmbH, Owen, Germany) and the first five trials within the range of $1.6-2.0 \mathrm{~m} / \mathrm{s}$ were included in the study. A similar approach was used for slow running and for fast running, in which the target speed was set at $3.6 \mathrm{~m} / \mathrm{s}$ and $4.3 \mathrm{~m} / \mathrm{s}$, respectively. Similar to the fast walking task, five successful trials were selected based on a maximum deviation of $0.2 \mathrm{~m} / \mathrm{s}$.

Kinematic data were collected using a Vicon Nexus ten MX T-Series camera system at $240 \mathrm{~Hz}$ (VICON, Oxford Metrics, Oxford, UK). Markers were placed on anatomical landmarks as defined by Plug-in Gait. Ground reaction forces and sagittal plane joint angles were extracted, resampled over a full gait cycle from each trial, and ensemble averaged to obtain a mean movement cycle and standard deviation. Additional data on slow walking were obtained from the literature. $^{23}$ All data were collected without any masses attached to the limbs.

\section{Optimal control approach}

Predictive simulations were carried out using an optimal control approach. ${ }^{26}$ Specifically for this project, system state trajectories $\mathbf{x}(t)$ and control trajectories $\mathbf{u}(t)$ were sought, which satisfy the system dynamics (4) as well as the task constraints of symmetry, periodicity and speed (Table 2), such that the following objective function is minimized

$$
\begin{aligned}
F(\cdot)= & \frac{1}{11}\left[\frac{1}{T} \sum_{i=1}^{10} \int_{0}^{T}\left(\frac{s_{i}(\mathbf{x}(t))-m_{i}(t)}{\sigma_{i}}\right)^{2} \mathrm{~d} t+\left(\frac{T-m_{T}}{\sigma_{T}}\right)^{2}\right] \\
& +\frac{W}{16 T} \sum_{i=1}^{16} \int_{0}^{T} u_{i}(t)^{2} \mathrm{~d} t
\end{aligned}
$$

in which $m_{i}(t)$ are measured trajectories of ten variables: three joint angles and the horizontal and vertical ground reaction forces, in each limb. The corresponding simulated variables in the model are $s_{i}$, which are either state variables (joint angles) or functions of the system state (ground reaction forces). Because of periodicity and symmetry, only half of a movement cycle needs to be simulated, with duration $T$, which may be different from its corresponding measured value $m_{T}$. As described in more detail elsewhere, ${ }^{22}$ the task constraints of symmetry and periodicity were represented by

$$
\mathbf{x}(T)=\mathbf{x}^{*}(0)+v T \mathbf{e}_{1}
$$

where $v$ is the prescribed walking or running speed, $\mathbf{x}^{*}$ represents the mirror image of $\mathbf{x}$, and $\mathbf{e}_{1}$ is the statespace unit vector for forward translation. The controls u were bound-constrained between zero (no muscle excitation) and one (full muscle excitation). The states $\mathbf{x}$ were not constrained.

The cost function (5) with two quadratic terms is commonly used in classical control theory, e.g. linear quadratic regulator design. The first "tracking" term in (5) will encourage the model to stay close to the desired movement, and the second "effort" term will encourage it to use its muscles efficiently. The five variables (two forces and three angles) that were used in the tracking term were selected because they are easy to measure and often reported in the literature. The difference between simulation and measurement is normalized to the between-trial standard deviations, $\sigma_{i}$, in each variable, making it dimensionless and emphasizing those variables, which are most reproducible in the human data. This is statistically equivalent to a maximum likelihood criterion for normally distributed measurements.

When mass is added to the limbs, the original movement will require more effort and the optimization will find a new, adapted, movement $\mathbf{x}(t)$ that is optimal for the new condition. The sensitivity of this adaptation depends on the weighting factor $W$ in the optimization objective (5). When $W$ is zero, the model does not care about effort and will not adapt its movement. When $W$ is very large, the model does not care about emulating the movement of the human participant and will simply find the movement that theoretically has the lowest effort for that running speed, as in Ackermann and van den Bogert. ${ }^{22}$ While the latter is attractive because it is entirely predictive and not dependent on data, it is our experience that this can lead to unrealistic predictions, such as extremely high impact forces and early knee flexion during stance. ${ }^{22}$ It is, therefore, hypothesized that an intermediate value of $W$, still to be determined, will produce realistic predictions of adaptive behavior.

\section{Solution method and protocol for simulation experiments}

The optimal control problem was transcribed into a large-scale nonlinear program (NLP) using direct collocation (DC). ${ }^{27}$ The continuous state and control trajectories were discretized in time, defining temporal sequences of states $\left(\mathbf{x}_{1}, \mathbf{x}_{2}, \ldots \mathbf{x}_{N}\right)$ and controls $\left(\mathbf{u}_{1}, \mathbf{u}_{2}\right.$, $\ldots \mathbf{u}_{N}$ ) where $N$ is the number of time samples (nodes) used to represent the trajectories. Because there are 50 
state variables and 16 control variables, as well as an unknown movement duration $T$, the total number of unknowns was $66 N+1$, and these are collected into a large vector, $\mathbf{X}$. Bounds were imposed on the controls within $\mathbf{X}$, which were not allowed to be lower than zero (no muscle activation) or larger than one (full muscle activation). Additional constraints on $\mathbf{X}$ arise from the task constraints of symmetry, periodicity, and running speed, as well as from the discretization of the system dynamics (4). A midpoint Euler discretization was used, which replaces the implicit differential equation (4) by an algebraic constraint for each pair of adjacent nodes:

$$
\mathbf{f}\left(\frac{\mathbf{x}_{i+1}+\mathbf{x}_{i}}{2}, \frac{\mathbf{x}_{i+1}-\mathbf{x}_{i}}{t_{i+1}-t_{i}}, \frac{\mathbf{u}_{i+1}+\mathbf{u}_{i}}{2}\right)=0
$$

The optimal control problem now has to be transformed into a standard NLP form: minimize the objective function $F(\mathbf{X})$, subject to a finite number of constraints $C_{i}(\mathbf{X})=0$ and bounds $\mathbf{L} \leqslant \mathbf{X} \leqslant \mathbf{U}$.

To solve this NLP, two solvers were used that are available for Matlab. IPOPT is an interior point (IP) method, ${ }^{28}$ which was used to find an initial solution on a coarse mesh ( $\operatorname{small} N$ ) when no initial guess was available. Subsequent optimizations, using previous results as an initial guess, were obtained with the SNOPT solver (Tomlab Optimization, Seattle, WA), which is an implementation of a sparse sequential quadratic programming (SQP) algorithm. ${ }^{29}$ Consistent with recommendations by Betts, ${ }^{27}$ the SQP method was found to be the most robust, while the IP method required far more iterations and often lost convergence even with a good initial guess. On the other hand, only the IP method could solve the problem when no initial guess was available, albeit on a coarse mesh.

Solutions were first obtained for the baseline musculoskeletal model without added masses, using slow walking data ${ }^{23}$ in the tracking term of the optimization objective. First, successive mesh refinement was performed to determine the number of nodes $N$ that was needed to obtain sufficiently accurate solutions. This solution was also used to evaluate the model in comparison to human testing data. Second, optimizations were performed with the fast walking data to determine a suitable effort weighting factor, $W$. Finally, these values of $N$ and $W$ were used in optimizations for all four movements, with the baseline model and with additional masses of $200 \mathrm{~g}$ and $400 \mathrm{~g}$ placed at one of four locations: knee, mid-shank, ankle, and mid-foot. The magnitude of the masses was selected based on pilot tests which showed that larger masses are not practical or comfortable. Four locations for the masses were selected to avoid an a priori assumption that the most distal placement would generate the optimal training effect, and perhaps more importantly, to demonstrate how a large design parameter space can be explored via modeling. Care was taken to start each new optimization from a previous optimization result that was close enough to ensure convergence of the SNOPT solver to an optimum in the same basin of attraction.

\section{Outcome measures}

For the purpose of model evaluation, time histories of simulated joint angles and ground reaction forces will be presented, so that they can be compared to the corresponding variables in the human studies from the literature.

Energy cost of the movement was used as the primary quantitative outcome variable for the simulation experiments with added limb mass because this can be compared to metabolic measurements that have been reported in the literature. Energy cost was calculated as the positive mechanical work done by the muscle fibers, ${ }^{30}$ and expressed as work done per unit time, that is, average power. For each muscle, this work rate is calculated as follows from the force and lengthening velocity in the contractile element

$$
P=\frac{1}{T} \int_{0}^{T}\left[-F_{C E}(t) \dot{L}_{C E}(t)\right]^{+} \mathrm{d} t
$$

where $[\mathrm{x}]+$ equals $\mathrm{x}$ when $\mathrm{x}$ is positive and zero when $\mathrm{x}$ is negative. These values are then summed over the 16 muscles in the model.

A secondary outcome variable is the stride length, which was defined as the prescribed speed $v$ multiplied by the predicted gait cycle duration $2 T$. This variable was chosen because it is the only kinematic variable that has been reported as being significantly affected by added limb mass in human participants. ${ }^{11}$

Finally, time histories of predicted muscle force patterns are presented for specific experiments with added limb mass, to demonstrate the potential for predicting training effects on certain muscles.

\section{Results}

Figure 2 shows the results of the mesh refinement experiments, using the slow walking data in the optimization objective, with effort weighting $W=10$. The error in predicted energy cost, due to temporal discretization, was less than $0.1 \%$ for a 50 -node mesh. Based on these results, it was decided to use $N=50$ for all subsequent work. The 50-node solution is shown in Figure 3, showing excellent tracking for all five measured variables and efficient phasic muscle activation patterns that are consistent with electromyographical recordings during normal gait. ${ }^{31}$ This qualitative evaluation of the model shows that the model can replicate human performance with sufficient realism.

Mechanical energy measures for fast walking, predicted with a wide range of effort weight factors $W$, are presented in Figure 4. When $W$ is very small, the model is willing to spend excessive and unrealistic amounts of effort to maintain its tracking of the original movement. 


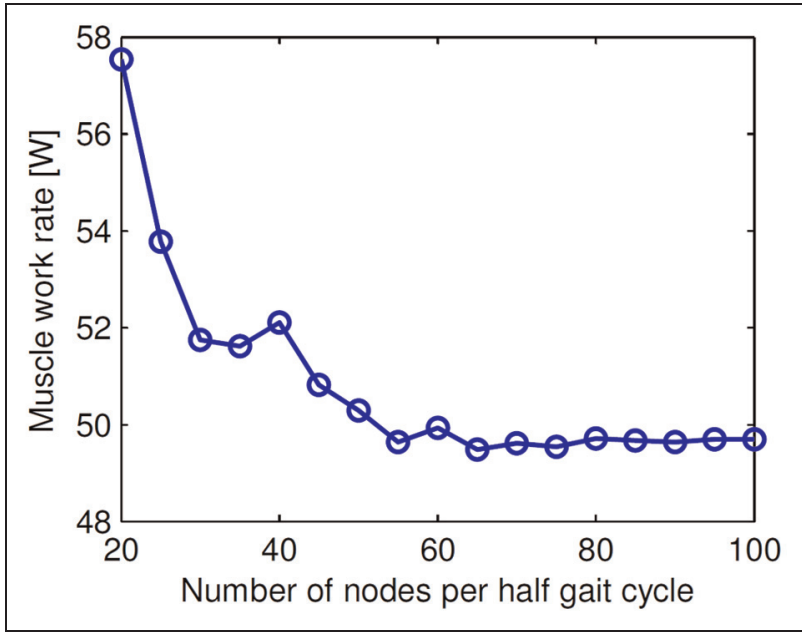

Figure 2. Energy cost predictions from optimal control solutions for slow walking $(1.10 \mathrm{~m} / \mathrm{s})$ at effort weighting $W=10$, with increasing number of time nodes in the temporal mesh.

This results in muscle activation patterns with excessive co-contraction and noise, causing increased energy cost. At very large values of $W$, the model will become overly concerned with effort and no longer attempts to track the original movement. Fortunately however, the relative increase in energy cost due to added mass on the ankle, predicted with intermediate values of $W$, was largely independent of $W$, especially below $400 \mathrm{~g}$ of added mass which is the main interest of the study. These results also show that the increase in energy cost is proportional to the amount of added mass, which is consistent with physiological studies. ${ }^{9-11}$ Based on these results, a medium value of $W=10$ was selected for the final part of the study. It was observed that with this weighting, the tracking and effort contributions in the optimization objective (5) were approximately equal, for all movements studied. This ensured that neither term dominated the optimization process, and consequently, generated predicted movements that do not stray far from the desired movement (tracking) with smooth and efficient muscle control patterns (effort).

Simulated movements for the four different speeds, without added limb mass, are shown in Figure 5, and the effects of the added limb mass on energy cost are presented in Figure 6. The effects were proportional to the amount of added mass, and generally largest when mass was added to the foot or ankle.

Predicted mechanical effects of the added masses were generally small. Stride length always increased with added mass, as shown in Figure 7 for the case of slow running. Other changes in kinematics and ground

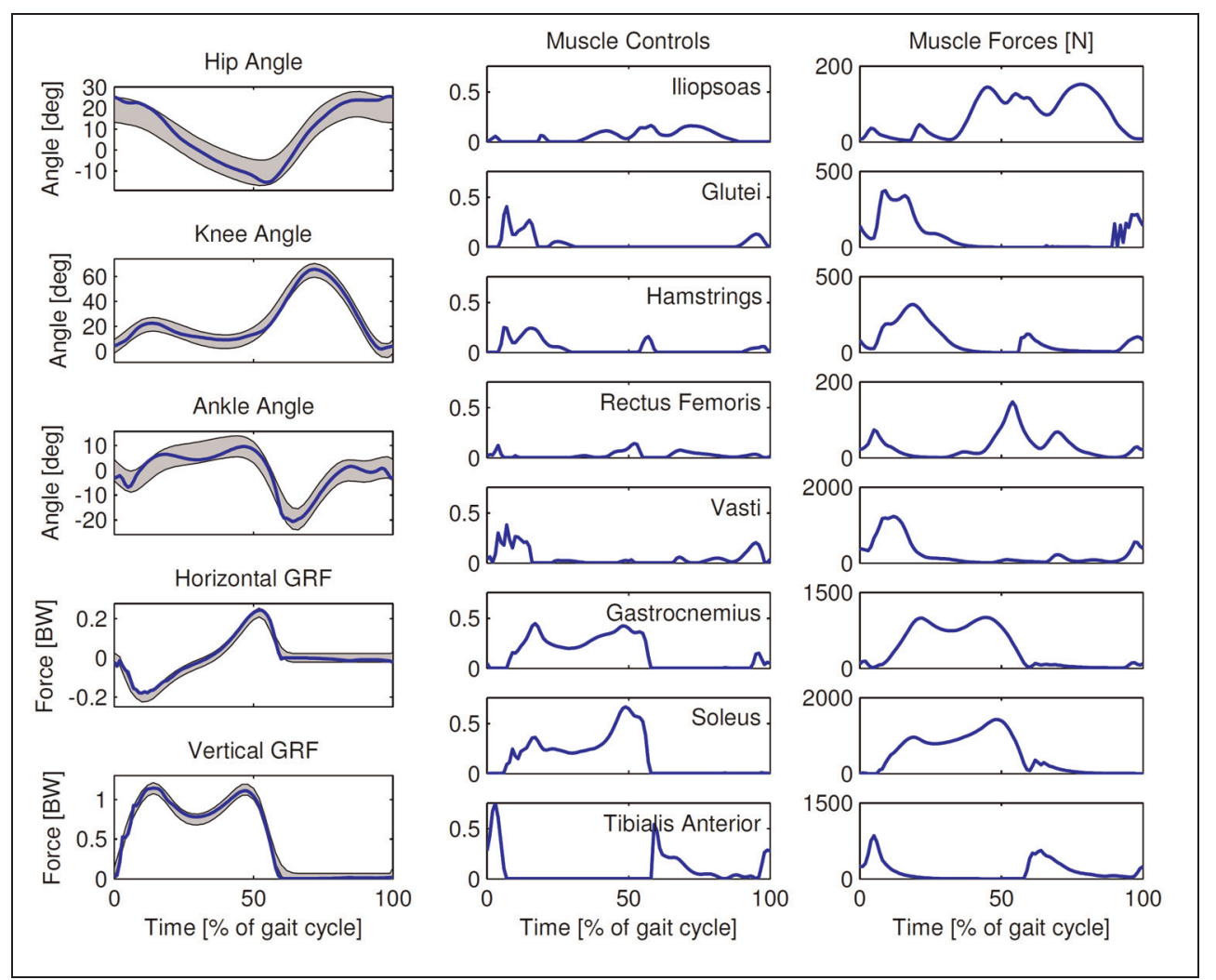

Figure 3. Optimal control solution for slow walking $(1.10 \mathrm{~m} / \mathrm{s})$. Solid curves are results from the model, shaded areas are the mean and standard deviation of human gait data that was used in the tracking term of the optimization objective. GRF: ground reaction force. Joint angles are flexion angles, defined relative to a standing posture in which all body segment reference frames are aligned with the global reference frame (Figure I). All horizontal axes represent a full gait cycle. Note the different vertical scales in the muscle force plots. 


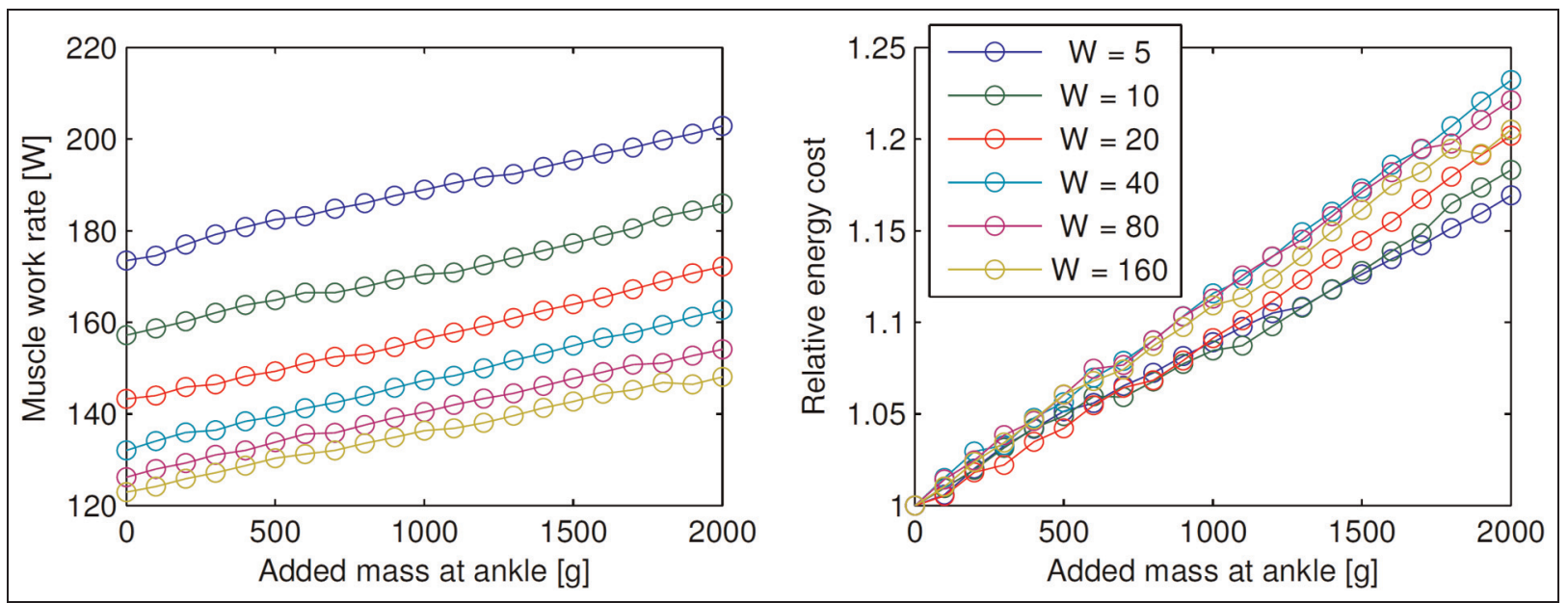

Figure 4. Energy cost for fast walking with added mass at the ankle, predicted with various effort weight factors (W) in the optimization objective. The left graph shows the actual energy cost, the right graph shows changes relative to the baseline model with no attached mass.

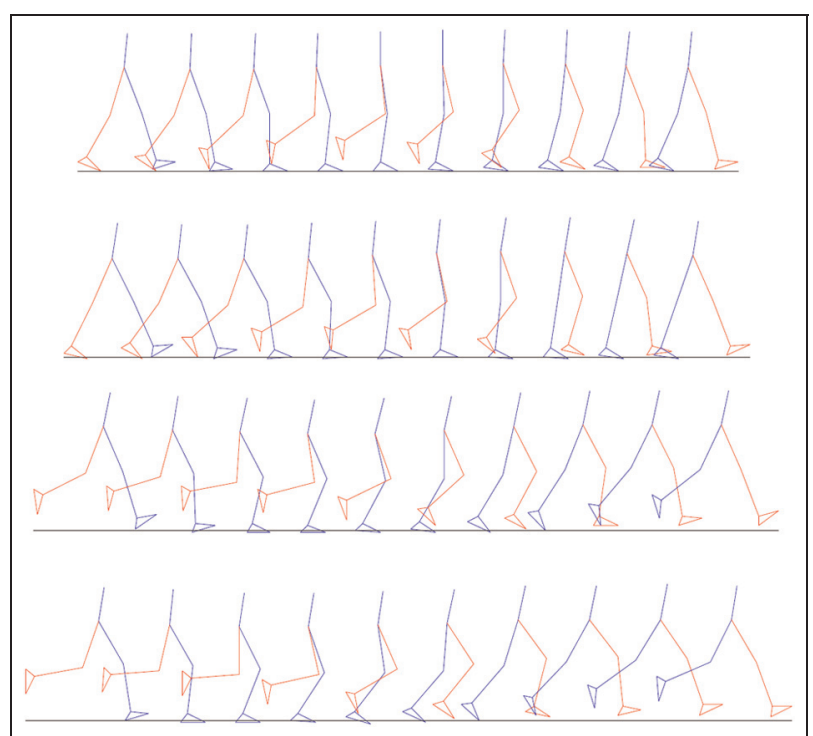

Figure 5. Simulations of the four movements, without added limb mass. Half of a movement cycle is shown, from heel strike to contralateral heel strike. From top to bottom: slow walking $(1.10 \mathrm{~m} / \mathrm{s})$, fast walking $(1.82 \mathrm{~m} / \mathrm{s})$, slow running $(3.60 \mathrm{~m} / \mathrm{s})$, and fast running $(4.27 \mathrm{~m} / \mathrm{s})$.

reaction forces were evaluated qualitatively and not large enough to merit specific attention. Potential muscle training effects were examined in the condition that had the largest metabolic effect, fast running with $400 \mathrm{~g}$ mass attached to the foot (Figure 8). In this condition, there were substantial increases in peak load in the hamstrings group $(+26 \%)$ and in the rectus femoris $(+17 \%)$.

\section{Discussion}

In this study, an optimal control approach was developed which allows a musculoskeletal model to predict the effect of mechanical interventions on human performance. The method was used to predict the effect of a mass added to the limbs on the energy cost, kinematics, and muscle recruitment during walking and running. The baseline model was able to replicate a human participant's walking and running movements, and it used control patterns that are consistent with normal human EMG. When mass was added, the optimal control method predicted specific changes in neuromuscular control, limb kinematics, and energy cost. Predictions were consistent with experimental human data from the literature, when available.

The predicted energetic effects of added mass were in agreement with the exercise physiology literature. The most commonly studied condition is running with added weight on the foot, where the model predicted an increase in energy cost of $0.74 \%$ per $100 \mathrm{~g}$ of added mass at $3.60 \mathrm{~m} / \mathrm{s}$ and $0.86 \%$ at $4.27 \mathrm{~m} / \mathrm{s}$ (Figure 4). Human oxygen uptake studies found increases in energy cost, per $100 \mathrm{~g}$ added mass, of $1.33 \%$ at $2.68 \mathrm{~m} / \mathrm{s},{ }^{12}$ $0.72 \%$ at $3.33 \mathrm{~m} / \mathrm{s}$, ${ }^{11}$ and $0.8 \%{ }^{13}$ and $0.86 \%{ }^{9}$ at unspecified speeds. For slow walking, the increases in energy cost were small, and interestingly, the shank-ankle area seemed to be a "sweet spot" where addition of mass does not require as much additional muscle work as when placing the same mass on the thigh. The limb swing at this speed may be a mostly passive pendular movement that is unaffected by additional mass as long as the center of mass remains in the same location. For fast walking, the increase in energy cost was much larger and, in relative terms, as large as for fast running. This walking speed was well above typical preferred walking speeds, requiring a rapid limb swing that is actively powered by muscles, and is, therefore, more sensitive to added mass.

Early attempts at estimating the energy cost of running with added mass have been based on variations of the total (kinetic plus potential) energy of the added mass during the gait cycle. ${ }^{11}$ These calculations 


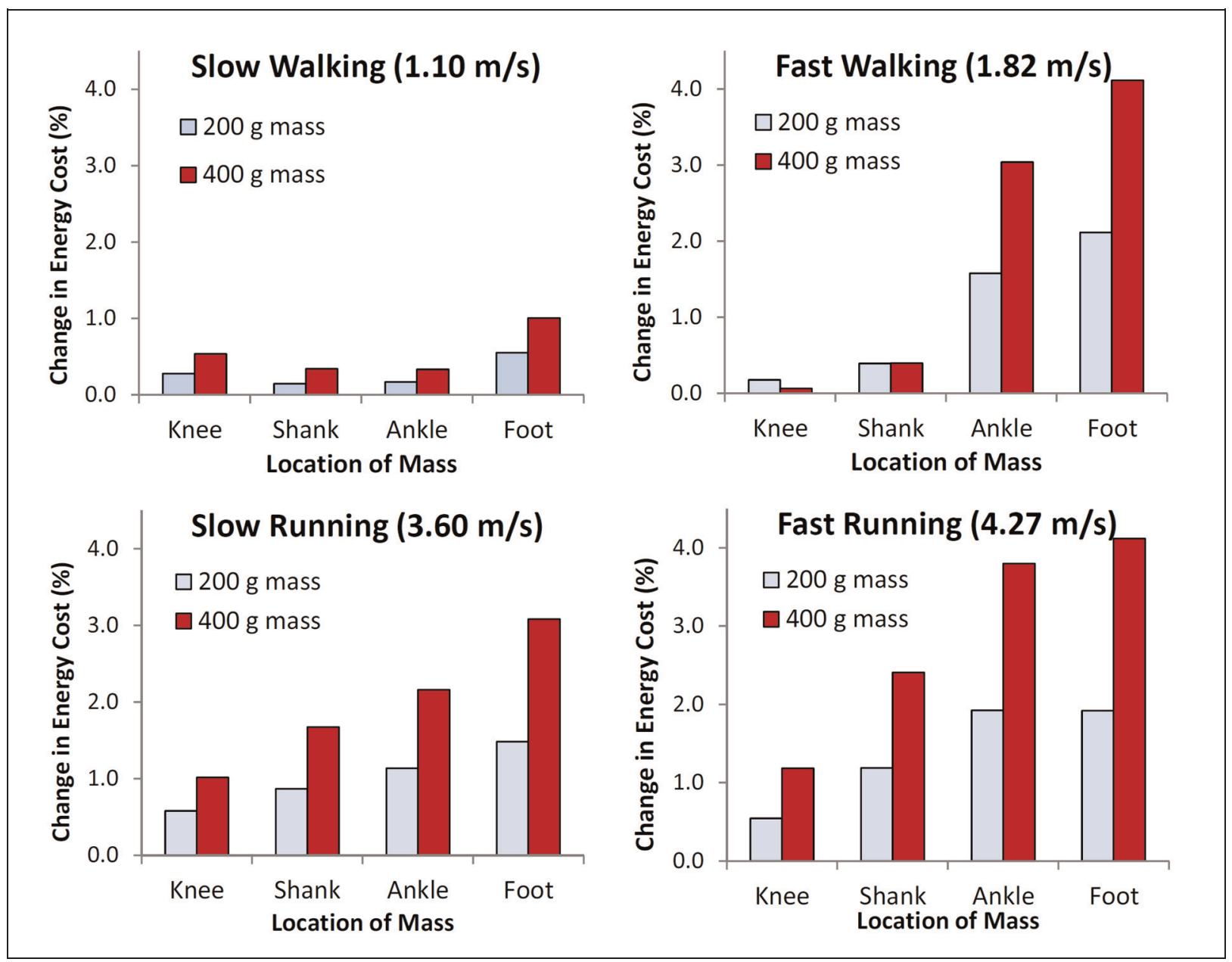

Figure 6. Predicted effects of added mass on the energy cost of walking and running.

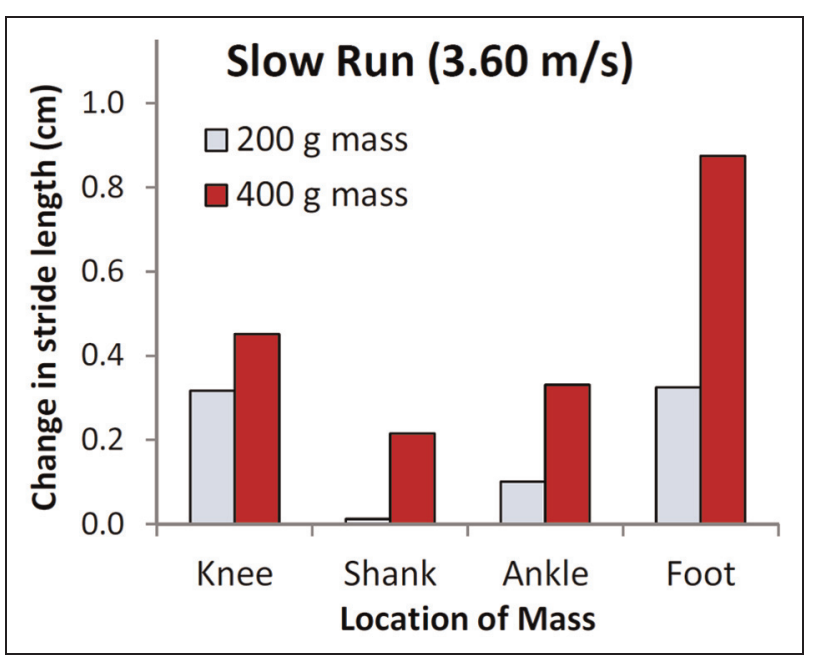

Figure 7. Predicted effect of added mass on stride length during running at $3.60 \mathrm{~m} / \mathrm{s}$.

typically overestimate the metabolic cost of the added weight by a factor of three, ${ }^{11}$ and this was also seen when such calculations were done on the human motion data in this study. These simple methods may overestimate the energy cost because not all changes in energy of the attached mass can be attributed to muscle activity and metabolic cost. Total energy of the added mass can fluctuate due to passive energy transfer between the segments in a multilink pendulum, and due to storage and release of elastic energy. Neither of these mechanisms is associated with metabolic cost. A full musculoskeletal dynamics model, as used here, includes these mechanisms and can therefore produce a lower, and better, estimate of the metabolic cost of added mass during walking and running.

It should be noted that the energy cost calculations were based on the mechanical energy of muscle contraction in the model and no attempt was made to estimate the metabolic energy used by muscles. While the mechanical and metabolic energy are likely correlated, especially for the relatively small changes induced by the added weights, their magnitudes may well be very different. Muscle models that predict metabolic energy have been developed ${ }^{32}$ and could be considered in further studies.

The prediction of increased stride length during running with added foot mass (e.g. $1.1 \mathrm{~cm}$ increase for $400 \mathrm{~g}$; Figure 7) was consistent with a human study that reported a stride length increase of $1.4 \mathrm{~cm}$ with a $500 \mathrm{~g}$ added mass at similar running speed. ${ }^{11}$ This is likely a 


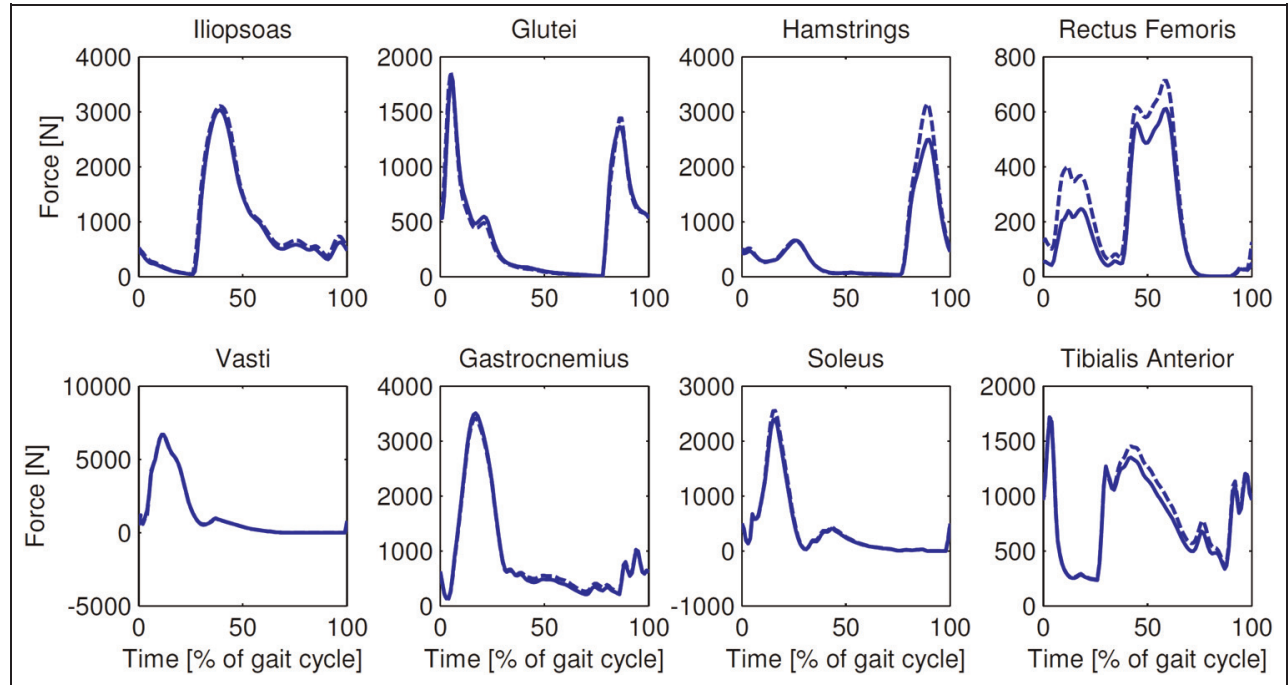

Figure 8. Muscle forces during fast running $(4.27 \mathrm{~m} / \mathrm{s})$, without added mass (solid curves) and with a $400 \mathrm{~g}$ mass attached at each ankle (dashed curves).

result of the increased moment of inertia of the limb, which reduces the natural frequency of the swing movement. Further confirmation comes from observations on an amputee runner, with reduced mass below the knee, who ran with much shorter strides than ablebodied runners. ${ }^{8}$ There is one other human study that reports kinematic and kinetic effects of added mass, ${ }^{10}$ but this was for walking with extremely large foot masses of 4 and $8 \mathrm{~kg}$, which is not comparable to the simulated conditions in the present study. Perhaps related to the increased moment of inertia and stride length, it was also predicted by the model that added mass causes a substantial increase in force in the biarticular thigh muscles (Figure 8). This is a surprisingly large muscle-specific training effect, considering that each limb was weighted by only $0.5 \%$ of the total body mass. The model apparently preferred to recruit biarticular muscles rather than one-joint muscles, possibly because these muscles can produce the required increase in hip and knee joint moments at a lower muscle activation cost (the second term in equation (5)). Human tests with EMG or inverse dynamic analysis should be performed to help confirm the predicted training effects.

The musculoskeletal model was two dimensional and had some important limitations. There was no motion of the pelvis in the frontal plane, no foot pronation, and no "wobbling mass" in the model. These features had been included in a previous running model, ${ }^{33}$ and helped avoid excessive impact forces. Wobbling mass alone will attenuate impact even in a planar model. ${ }^{34}$ In the present work, it seems that the optimal control method was able to fine tune the movement and muscle actions to achieve sufficiently soft landings even without wobbling mass. This likely did not affect the results of the present study which was mainly about muscle function, but a more realistic three-dimensional model with wobbling mass should be considered for future predictive simulations of running, especially when applied to injuries where the impact response must be represented accurately. Initial experience with a three-dimensional walking model ${ }^{35}$ has shown that the optimal control approach still works, but at enormous computational expense due to the higherdimensional state and control spaces. No attempt was made to customize the model based on the body segment parameters and muscle properties of the participant from whom the movement data were obtained. It is not expected that this would have affected the results, but this remains to be verified in a future study with multiple participants. It is expected, however, that results will be somewhat sensitive to the participant's style of running, which the model will replicate. It is recommended that multiple participants are used in future studies, such that statistical analysis can be done on the model predictions, exactly as in human research studies. $^{5}$

The optimal control approach for predictive simulation was based on the assumption that adaptive human behavior is governed by a desire to stay close to the original movement and minimize effort. Furthermore, these two goals were weighted so that they contributed roughly equally to the overall optimization objective as defined by equation (5). While this assumption produced certain predictions that agreed very well with observations, it would be desirable to design human experiments that specifically test the validity of this optimization criterion. Specifically, it must be noted that the predicted changes in muscle recruitment may be dependent on the particular choice of effort term in the cost function, and for these predictions there are as yet no corresponding observations in humans.

The numerical methods that were used to solve the optimal control problem worked well, but care must be taken that the initial guess for solving a new problem is already close enough to the solution. This is typically 
accomplished by solving a sequence of related problems as was done in the present study. For the very first optimization problem, to simulate slow walking without attached masses, it was difficult to obtain convergence. Even after convergence, the solution was sometimes obviously a local optimum, recognized by lack of realism and unusually high value of the optimization objective (5). These challenges were overcome by repeated attempts with manually generated initial guesses to obtain the very first result. Subsequent optimizations, such as mesh refinements, increased walking speed, or added weight, always were done in a sequence such that a close initial guess was available. These optimizations occasionally converged a local minimum that was easily recognizable. It must be noted there never was a local optimum that was not easily recognized by lack of realism and high value of the cost function. The correct solution could always be obtained by making the changes in model or task small enough. For instance, when added mass was increased in steps of $200 \mathrm{~g}$, there were occasional convergence problems. In steps of $100 \mathrm{~g}$, this no longer happened. Each new solution required 5-10 min of computation time each, on a $2 \mathrm{GHz}$ Intel T2500 processor. This is fast enough to allow effective and possibly interactive exploration of the design space.

A key feature of the optimal control approach is that the movements and forces produced by the human body are not considered as given, but are allowed to adapt to the equipment, either taking advantage of equipment properties, or compensating for shortcomings in the design. These methods may also be combined with detailed finite element (FE) models of the equipment being studied. FE simulations of sports equipment are currently performed with boundary conditions that represent a given human movement that does not adapt. Humans, however, are known to adapt their movement, and this is likely an important mechanism through which sports equipment can affect performance and injury risk. ${ }^{4}$ Predictive simulations that straddle the domains of FE modeling and multibody modeling, and include neuromuscular adaptive behavior, are numerically challenging, ${ }^{36}$ but will eventually be solvable and integrated in a computer-aided design process for sports equipment.

It was demonstrated in this paper that, for a specific equipment design question, predictive musculoskeletal simulation can be done in less time and at lower cost than human experiments, while producing essentially the same results. This allows the designer to obtain a more complete "map" of the design parameter space (e.g. Figure 6). For questions related to maximal performance or injury, human testing is even more difficult and predictive modeling is especially attractive. It must be kept in mind, however, that computational models will never fully represent all aspects of human mechanics, physiology, and behavior. The required level of modeling detail is not always known, and may depend on the questions for which the model is used. We therefore suggest that simulation should be used for an initial exploration, in order to select promising designs for prototyping and human testing. Further work is still needed to expand both the validity and practicality of this approach. Specifically, optimality criteria must be evaluated through human testing, more realistic models must be developed, and faster and more robust numerical methods are needed to solve optimal control problems on those models.

\section{Conclusions}

The main conclusions from this work were as follows.

1. A computational musculoskeletal model with optimal control can be used to predict how human performance is affected by a change in the mechanical system. With a planar musculoskeletal model, the computation time is short enough that a large design parameter space can be effectively explored.

2. When the method was applied to the question of walking and running with attached weights, the predicted metabolic effects were consistent with literature data from human experiments.

3. The model-based predictions suggested musclespecific training effects of attached weights, most prominently in the biarticular thigh muscles during fast running.

\section{Funding}

This research received no specific grant from any funding agency in the public, commercial, or not-for-profit sectors.

\section{References}

1. Nigg BM, Bahlsen HA, Luethi SM, et al. The influence of running velocity and midsole hardness on external impact forces in heel-toe running. $J$ Biomech 1987; 20: 951-959.

2. Wright IC, Neptune RR, van den Bogert AJ, et al. Passive regulation of impact forces in heel-toe running. Clin Biomech 1998; 13: 521-531.

3. Hardin EC, van den Bogert AJ and Hamill J. Kinematic adaptations during running: effects of footwear, surface, and duration. Med Sci Sports Exerc 2004; 36: 838-844.

4. Frederick EC. Kinematically mediated effects of sport shoe design: A review. J Sports Sci 1986; 4: 169-184.

5. McLean SG, Huang $X$ and van den Bogert AJ. Investigating isolated neuromuscular control contributions to non-contact anterior cruciate ligament injury risk via computer simulation methods. Clin Biomech 2008; 23: 926-936.

6. Gambaryan PP. How mammals run. New York: John Wiley \& Sons, 1974.

7. Catlin MJ and Dressendorfer RH. Effect of shoe weight on the energy cost of running. Med Sci Sports 1979; 11: 80 . 
8. Weyand PG and Bundle MW. Point: Artificial limbs do make artificially fast running speeds possible. $J$ Appl Physiol 2010; 108: 1011-1012.

9. Bhambhani Y, Burnham R, Singh M, et al. Ankle and wrist weights: their effect on physiologic responses during treadmill running. Arch Phys Med Rehabil 1989; 70: 291296.

10. Browning RC, Modica JR, Kram R, et al. The effects of adding mass to the legs on the energetics and biomechanics of walking. Med Sci Sports Exerc 2007; 39: 515-525.

11. Martin PE. Mechanical and physiological responses to lower extremity loading during running. Med Sci Sports Exerc 1985; 17: 427-433.

12. Meyers MJ and Steudel K. Effect of limb mass and its distribution on the energetic cost of running. J Exp Biol 1985; 116: 363-373.

13. Miller JF and Stamford BA. Intensity and energy cost of weighted walking vs. running for men and women. $J$ Appl Physiol 1987; 62: 1497-1501.

14. McArdle WD, Katch FI and Katch VL. Energy expenditure at rest and during physical activity. In: McArdle WD, Katch FI, Katch VL (eds) Essentials of exercise physiology, Volume 1. 3rd ed. Philadelphia, PA: Lippincott Williams and Wilkins, 2006, pp.260-289.

15. Kadaba MP, Ramakrishnan HK, Wooten ME, et al. Repeat Ability of kinematic, kinetic, and electromyographical data in normal adult gait. J Orthop Res 1989; 7: 849-860.

16. Albertus-Kajee Y, Tucker R, Derman W, et al. Alternative methods of normalising EMG during running. $J$ Electromyogr Kinesiol 2011; 21: 579-586.

17. Hamner SR, Seth A and Delp SL. Muscle contributions to propulsion and support during running. $J$ Biomech 2011; 43: 2709-2716.

18. Gerritsen KGM, van den Bogert AJ and Nigg BM. Direct dynamics simulation of the impact phase in heeltoe running. J Biomech 1995; 28: 661-668.

19. Gerritsen KGM, Nachbauer W and van den Bogert AJ. Computer simulation of landing movement in downhill skiing: anterior cruciate ligament injuries. $J$ Biomech 1996; 29: 845-854.

20. Hardin EC, Su A and van den Bogert AJ. Pre-impact lower extremity posture and brake pedal force predict foot and ankle forces during an automobile collision. $J$ Biomech Eng 2004; 126: 770-778.

21. Gerritsen KGM, van den Bogert AJ, Hulliger M, et al. Intrinsic muscle properties facilitate locomotor controlA computer simulation study. Motor Control 1998; 2: 206-220.

22. Ackermann M and van den Bogert AJ. Optimality principles for prediction of human gait. $J$ Biomech 2010; 43: 1055-1060.
23. Winter DA. Biomechanics and motor control of human movement. 3rd ed. New York: John Wiley \& Sons, 2005.

24. Zajac FE. Muscle and tendon: properties, models, scaling, and application to biomechanics and motor control. Crit Rev Biomed Eng 1989; 17: 359-411.

25. McLean SG, Su A and van den Bogert AJ. Development and validation of a 3-D model to predict knee joint loading during dynamic movement. J Biomech Eng 2003; 125: 864-874.

26. van den Bogert AJ, Blana D and Heinrich D. Implicit methods for efficient musculoskeletal simulation and optimal control. Procedia IUTAM 2011; 2: 297-316.

27. Betts J. Practical methods for optimal control and estimation using nonlinear programming. 2nd ed. Philadelphia, PA: SIAM, 2010.

28. Wächter A and Biegler LT. On the implementation of a primal-dual interior point filter line search algorithm for large-scale nonlinear programming. Math Prog 2006; 106: 25-57.

29. Murray W, Gill PE and Saunders MA. SNOPT: An SQP algorithm for large-scale constrained optimization. SIAM J Optim 2002; 12: 979-1006.

30. Neptune RR, Zajac FE and Kautz SA. Muscle mechanical work requirements during normal walking: the energetic cost of raising the body's center-of-mass is significant. J Biomech 2004; 37: 817-825.

31. Hof AL, Elzinga H, Grimmius W, et al. Speed dependence of averaged EMG profiles in walking. Gait Posture 2002; 16:78-86.

32. Umberger BR, Gerritsen KG and Martin PE. A model of human muscle energy expenditure. Comput Methods Biomech Biomed Engin 2003; 6: 99-111.

33. Neptune RR, Wright IC and van den Bogert AJ. A method for numerical simulation of single limb ground contact events: application to heel-toe running. Comput Methods Biomech Biomed Engng 2000; 3: 321-334.

34. Pain MT and Challis JH. The influence of soft tissue movement on ground reaction forces, joint torques and joint reaction forces in drop landings. J Biomech 2006; 39: 119-124.

35. Ackermann MA and van den Bogert AJ. Simulation of gait using a 3D musculoskeletal model. American Society of Biomechanics annual meeting, Pennsylvania State University, 2009. Available at: http://www.asbweb.org/conferences/2009/pdf/1123.pdf

36. Halloran JP, Ackermann M, Erdemir A, et al. Concurrent musculoskeletal dynamics and finite element analysis predicts altered gait patterns to reduce foot tissue loading. J Biomech 2010; 43: 2810-2815. 